\title{
El Guiniguada.
}

(Revista de investigaciones y experiencias en Ciencias de la Educación)

eISSN: 2386-3374

Journal information, indexing and abstracting details, archives, and instructions for submissions: http://ojsspdc.ulpgc.es/ojs/index.php/ElGuiniguada/index

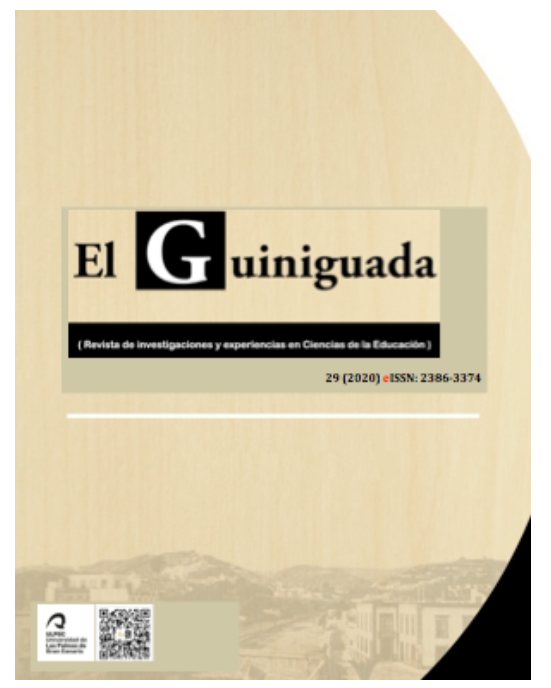

\section{Inventando la Heroína de las Mil \\ Caras: una propuesta didáctica de creación literaria}

Inventing the Heroine of a Thousand Faces: a Creative Writing Didactic Proposal

\section{Catalina Millán Scheiding} Berklee College of Music

DOI (en Metadatos y en Sumario Revista)

Recibido el 23/10/2019

Aceptado el 02/05/2020 


\title{
Inventando la Heroína de las Mil Caras: una propuesta didáctica de creación literaria \\ Inventing the Heroine of a Thousand Faces: a Creative Writing \\ Didactic Proposal
}

\author{
Catalina Millán Scheiding \\ Berklee College of Music \\ catalinamillanscheiding@gmail.com
}

\section{RESUMEN}

Esta propuesta didáctica ejemplifica el uso de la escritura creativa como una forma de acercarse al discurso de género, a través de la generación de un héroe o heroína de fantasía. Se ofrece una actividad en la que se trabaja el conflicto aparente y el conflicto subyacente, donde los roles de género pueden ofrecer respuestas diferentes y redefinir las estructuras narratológicas. El alumnado trabaja sobre su propia creación literaria para definir sus expectativas retóricas y los conflictos hegemónicos que se presentan en las historias de fantasía y ficción de su contexto social e ideológico. El contraste de los textos de creación propia con el análisis de textos y ejemplos audiovisuales de fantasía y ciencia ficción de creadoras literarias y de personajes femeninos presenta la oportunidad de generar un espacio contrastivo y constructivo, a la vez que enlaza con competencias educativas y facilita un acercamiento comparativo a la crítica literaria.

\section{PALABRAS CLAVE}

MATERIAL DIDÁCTICO, ESCRITURA CREATIVA, ROL DE LOS GÉNEROS

\begin{abstract}
This didactic proposal exemplifies the use of creative writing as a way to approach gender studies, through the creation of a fantasy hero. The activity offers the possibility of working both the apparent and underlaying conflicts, where gender roles can offer different answers and redefine narratological structures. The students work on their own literary creation to define their literary expectations and the predominant conflicts that appear in fantasy and fiction stories in their social and ideological context. The contrast of their own textual creations with the analyses of textual and audiovisual examples from fantasy and science fiction by female authors and including female characters offers the possibility of generating a contrastive and constructive space, which also links to educational competences and facilitates a comparatist approach to literary criticism.
\end{abstract}

\section{KEYWORDS}

INSTRUCTIONAL MATERIALS, CREATIVE WRITING, GENDER ROLES 


\section{INTRODUCCIÓN}

Existen innumerables variaciones del héroe de aventuras, que desde la tradición folclórica y mitológica occidental se han estudiado a través de la narratología (Propp, 1968; Bakhtin, 1981) o el comparatismo (Campbell, 1949). Sin embargo, aunque los datos subrayan un cambio de tendencia en la fantasía y el cómic (Shendruk, 2017), resulta difícil que el héroe sea una heroína y que, si lo es, ése no sea el factor principal que define la narración (Helford, 2000, 2016; Campbell, 2000). En esta propuesta didáctica, se pretende abrir la puerta a este tipo de cuestiones y generar un espacio de contraste y creación, desde donde se pueda acceder a la narrativa dominante de una forma constructiva y crítica.

El uso de textos de ficción para el desarrollo de las competencias interculturales está ampliamente probado y defendido (Burwitz-Melzer, 2001, p. 29-30) por su acercamiento subjetivo a la representación de valores y estereotipos. En esta propuesta se pretende dar un salto más amplio a esta actividad con el uso, no de un texto especifico, sino del carácter intertextual de los rasgos de género que se observan en narratología. Es decir, se pretende trabajar sobre la generalidad, en contra de propuestas enfocadas hacia la literatura creativa per se en el aula (García Carcedo, 2001, p. 52). De este modo, se pueden incorporar ejemplos literarios, pero también audiovisuales y participativos, donde tiene cabida el impacto o efecto de discursos que pueden considerarse alienados de la educación literaria general: cómics, fandom, videojuegos, juegos de mesa, adaptaciones audiovisuales, etc.

Arnot y Mac an Ghaill consideran que la conversación sobre género se presenta simplificada en los medios en general y, muy claramente, a lo largo de la escolarización obligatoria de los países occidentales (Arnot y Mac an Ghaill, 2006, p.1-2). Proponen que la construcción de la identidad personal y la individualización de la construcción biográfica conllevan un conflicto de identidad generalizada que se encuentra sobre todo en los roles del género femenino. El género femenino se presenta como el 'vencedor' de los movimientos neoliberales por su rotura con las responsabilidades que se le otorgan tradicionalmente (p. 5). Sin embargo, estudios educativos demuestran que los alumnos actuales conciben los roles de género como una compleja interacción entre la continuidad y los cambios sociales (p. 5) y su relación con otros factores y percepciones sociales como la clase, la raza o la etnicidad (p. 7). Es interesante considerar también lo que se denomina la crisis de la masculinidad en la educación y la tendencia actual a "re-masculinizar" el sistema educativo: Arnot y Mac an Ghill ponen como ejemplo a Reino Unido cuando hablan del éxito escolar ascendente del género femenino, mientras que en los últimos 20 años el campo semántico referido a la educación $y$, por consiguiente, la aplicación de diferentes políticas, trabaja con ideas bélicas y competitivas (p. 7-8). La discusión sobre cómo abordar estas cuestiones en el ámbito educativo está en constante dialéctica, y se aborda desde las políticas inclusivas oficiales hasta los estudios de propuestas didácticas que trabajan la inclusión y la diversidad, donde se introduce el tema de género (Leiva Olivencia et al, 2015). Mi intención es unirme a esta conversación con una propuesta didáctica que trabaja la escritura creativa, enfocando la atención en este ejemplo mayormente sobre los géneros binarios.

Se considera que el espacio creativo puede ofrecer un lugar seguro para el alumnado (Burwitz-Melzer, 2001, p. 42) y, dentro del contexto pedagógico, es interesante subrayar que el uso de textos de ficción enlaza diversas competencias educativas. A través de una actividad como la que se propone, se trabaja el aprendizaje situado 
(Lave y Wenger, 1991) donde el alumno puede utilizar sus predicciones sobre el hecho narrativo y compartirlos de forma activa en un contexto social, a la vez que se enlaza con la idea de las tareas de aprendizaje auténticas (Bruner, 1996, p. 44; Brown et al., 1989, p. 32), al estar ligados con los contextos culturales contemporáneos en que se encuentran inmersos los participantes. Quizá uno de los puntos más importantes a nivel pedagógico de este tipo de actividad sea el enfoque metacognitivo, ya que el objetivo principal es que el participante analice cómo ha adquirido un conocimiento y por qué (Pritchard, 1990, p. 27).

La idea de la propuesta didáctica se generó a través de un trabajo presentado por John Stephens sobre la reelaboración del final del 'Selkie Myth' (Stephens, 1992, p. 5967). Stephens presentaba su actividad en el contexto de la educación infantil y para trabajar el concepto de una doble lectura sobre el conflicto ideológico grupal que presenta el argumento del mito. Para detallar la actividad, resumo el argumento del mito: Los selkies son seres mágicos de la mitología escocesa que presentan aspecto humano, pero pueden vestirse con piel de foca y su naturaleza es la vida acuática. En el mito, un hombre encuentra una de las pieles de foca y, escondiendo la piel e imposibilitando su reconversión en foca, convierte a la mujer selkie a la que pertenece la piel en su esposa y madre de sus hijos. Un día, sin embargo, ella encuentra su piel y se marcha, abandonando el núcleo familiar. Por una parte, el conflicto aparente es el de la rotura del núcleo familiar, pero el conflicto subyacente es el de la subyugación obligada de la mujer al poder del hombre y la femineidad como inherentemente enlazada con procrear, por lo tanto, la 'desnaturalización' que se produce al abandonar a los hijos. La propuesta ejemplifica una de muchas actividades que parte de una historia ficticia y pide a los participantes que reescriban el final (BurwitzMelzer, 2001), con el objetivo de ilustrar la colisión que los participantes encuentran entre diversas ideologías y valores sociales.

La intención de la propuesta es incorporar la participación activa del alumno como generador de contenido, ya que trabajar la lectura desde la creación propia ilustraría más claramente los estereotipos narratológicos presentes. En mi caso, he usado este tipo de actividad en el aula para dirigirme tanto a temas de género como a temas de raza, con la elaboración de actividades específicas relacionadas. En este caso, la propuesta expuesta es la que aplico para iniciar la conversación sobre cuestiones de género en fantasía y ciencia ficción, pero puede ser modificada y adaptada según el nivel de los participantes y la competencia que tenga como objetivo el profesorado. Puesto que la actividad se propone en relación con el género, he incluido mayormente autoras de fantasía y ciencia ficción para los ejemplos narrativos; de este modo, no solo se propone una conversación sobre el género de los personajes, sino también de las creadoras del ámbito literario. La integración del discurso de género en el aula es un tema fundamental que enlaza con los Objetivos de Desarrollo Sostenible propuestos por las Naciones Unidas y, como se ha mencionado anteriormente, tiene un fuerte impacto en la construcción de subjetividades personales y globales, enlazado con las estructuras sociales, culturales y económicas. 


\section{PROPUESTA Y METODOLOGÍA}

\section{EL CONTEXTO EDUCATIVO}

La clase en la que tuvo lugar esta propuesta didáctica se llama Imaginary Lands (Tierras Imaginarias) y es uno de los cursos de literatura de segundo nivel que ofrece Berklee College of Music. Se puede cursar a partir de cumplir el prerrequisito de LENG 111, curso de Introducción a la Escritura Universitaria. Los objetivos de los cursos de segundo nivel incluyen el análisis del pensamiento critico y creativo a través del estudio literario y mediante la observación de las convenciones de género, y la aplicación de elementos de teoría literaria, además del análisis del impacto cultural, social e histórico que recibe y genera la literatura.

En Imaginary Lands estos objetivos se pretenden cumplir con ejemplos literarios y audiovisuales del ámbito de la fantasía y la ciencia ficción. Se incorporan conceptos teóricos de la literatura comparada, el dialogismo y el carnavalismo Bakhtiniano y la narratología. Al partir del terreno de la fantasía y la ciencia ficción, las competencias interculturales se trabajan desde la auto-conciencia hacia la escucha y adaptación propuestas por la UNESCO (Leeds-Hurwitz, 2010, p. 28) considerando la historia como un lugar donde generar empatía y una ventana hacia un mundo fantástico (Petit, 2002, p. 14) y donde las convenciones sociales pueden ser desconocidas y se puede construir una nueva subjetividad.

El caso que voy a presentar es la tercera variación de esta actividad didáctica y en ella se cuenta con 8 participantes, que tienen de 18 a 20 años. Todas las citas incluidas en la descripción de la actividad han sido generadas por los alumnos participantes. Considero que se podría poner en práctica esta propuesta con participantes que tengan de 12 años en adelante, ya que es cuando se estima que los niños y niñas entran en la etapa de las operaciones formales y pueden razonar de forma abstracta, deducir información de hipótesis y conectar con ideas que no forman parte de su experiencia personal (Messenger-Davis, 2010; Steinberg, 2005). También considero que se podría presentar como una actividad de introducción a estudiantes de magisterio del ámbito de la lengua y la literatura.

\section{EL DESARROLLO DE LA PROPUESTA PEDAGÓGICA}

La propuesta pedagógica se divide en cuatro fases diferenciadas: creación, información, re/creación y debate, que se dividen en un mínimo de dos sesiones, con un tiempo total que oscila entre la $1.30 \mathrm{~h}$ y las $2.00 \mathrm{~h}$. A nivel teórico, se pretende hacer un acercamiento a las funciones narratológicas propuestas por Propp, los opuestos binarios de Levi-Strauss y los estudios de argumento de Todorov.

\section{Actividad uno: creación}

A los participantes se les pide que generen un boceto para lo que podría ser un libro, película o videojuego de fantasía o ciencia ficción. Se les ofrece un listado de elementos a los que responder: protagonista, características principales (físicas y actitudinales), el lugar y el conflicto principal. Tienen un plazo de tiempo de 30 minutos en el aula en una actividad abierta en el portal de la clase donde trabajan a través de su ordenador. La actividad, en este caso, es individual.

\section{Objetivo}

El objetivo principal de la primera parte de la propuesta es tomar conciencia de sus predisposiciones culturales a la hora de acercarse a una historia de héroes y/o 
aventuras. Al acercarse a la creación sin un ejemplo o modelo específico, la intención es que los participantes tengan que trabajar sobre su propio bagaje intertextual a nivel argumental, referencial y estructural.

Resultados

En esta actividad, el resultado es el siguiente: el $87.50 \%$ definieron el género de sus personajes protagonistas, concebidos como héroes ( 7 de 8 ) y solo hay un personaje con un género indefinido, que pertenece al mundo de ficción 'Drag World' y que se describe como trans. De los protagonistas con un género definido, el porcentaje es el siguiente: $75 \%$ masculino, $12.50 \%$ femenino ( 1 de 8 ) y $12.50 \%$ trans ( 1 de 8 ).

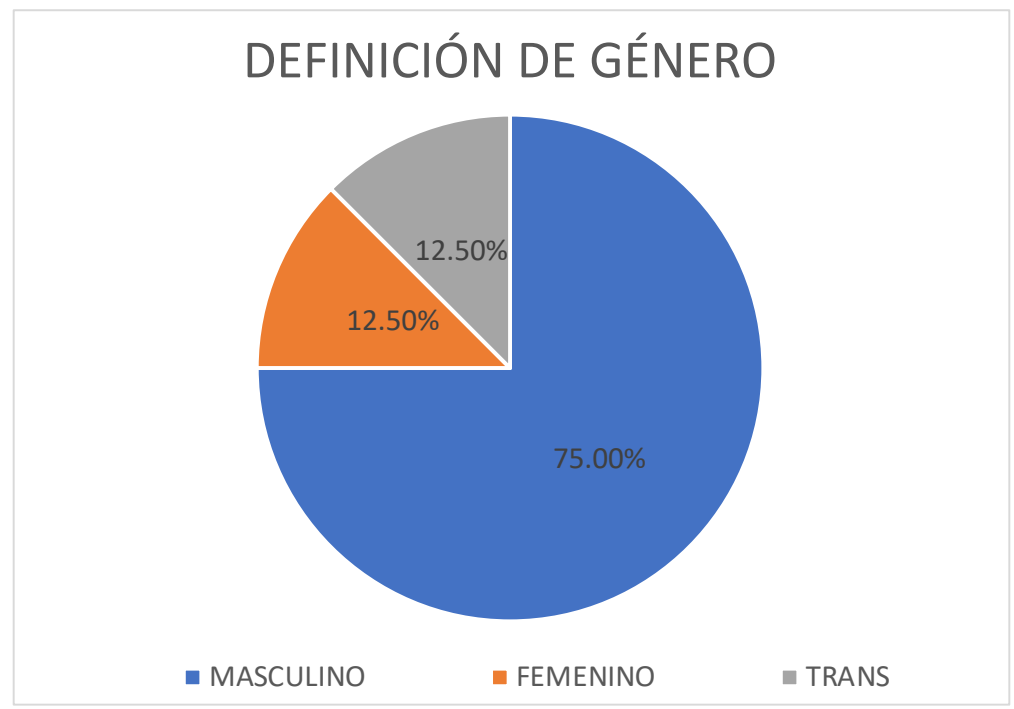

Figura 1: Definición de género de los personajes de la actividad uno

El ejemplo que propone un personaje trans es muy interesante, ya que la alumna presenta una batalla entre los personajes que tendrá lugar "exaggerating the characteristics of another Queen in a battle of wit. Whoever makes the most people laugh, wins" $"$. El conflicto, que aparece como una competición, ofrece una resolución no violenta.

Siete de ocho de los personajes protagonistas son descritos como jóvenes, el último 'de mediana edad'. Los poderes otorgados a los héroes son diversos, pero se dividen mayormente de la forma que ilustra la figura 2, con 'curación y luz' perteneciendo a la heroína femenina:

\footnotetext{
${ }^{1}$ En castellano: "exagerando las características de otra Reina en una batalla de ingenio. Quien haga reír a mayor cantidad de gente, gana."
} 


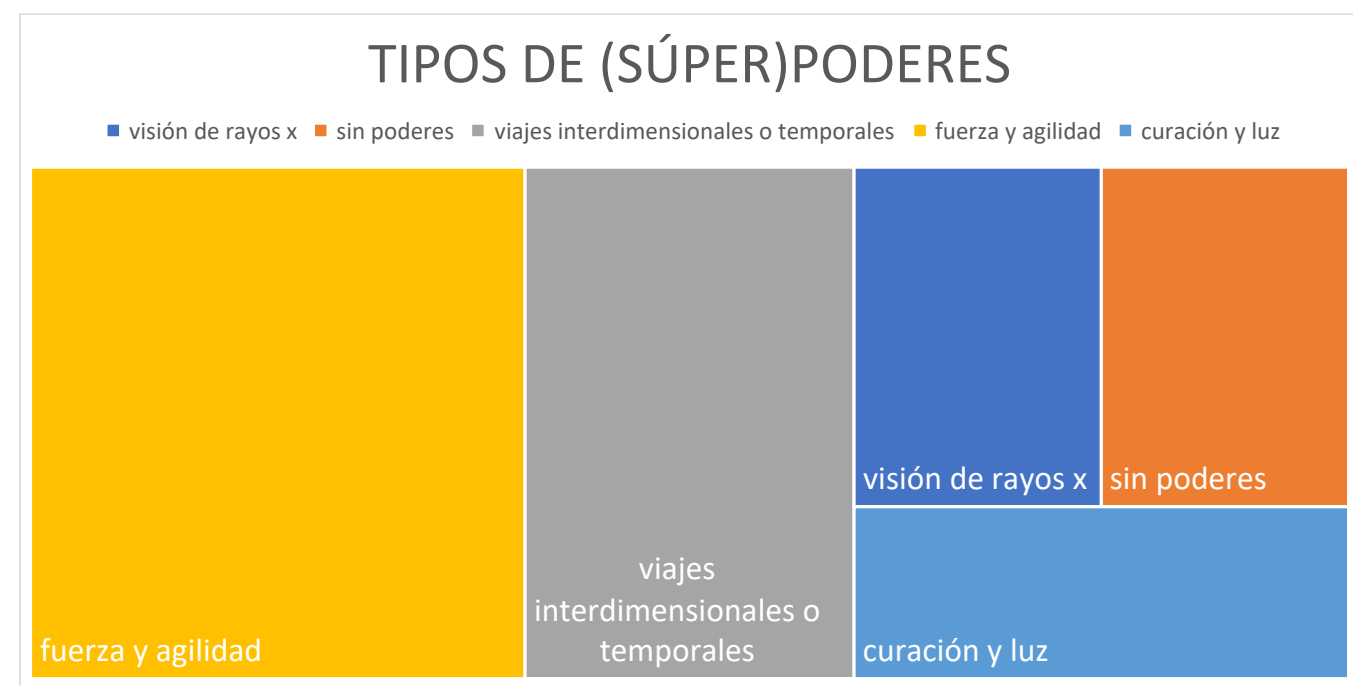

Figura 2: Tipos de poderes de los personajes de la actividad uno

Como motivación narratológica, encontramos ejemplos que usan la intertextualidad de historias fantásticas y de superhéroes como:

A teenage boy called Wade has the power of time travel. He discovered his powers when he was 5 years old and only his parents know about his gifts. Wade lives in a farm house in Michigan with his parents, who insist on Wade not using his powers. Wade has the urge to save people and protect people's fates ${ }^{2}$.

El relato se inspira en Superman y no explica con claridad la motivación hacia "hacer el bien".

También aparece un ejemplo que contempla el cambio climático, con la creación de un planeta (llamado Dukkha) donde una de las regiones está explotando los recursos naturales en detrimento del conjunto. El alumno presenta a "Djesse, a teenage human living in Manico $[\ldots]^{3}$ Djesse must choose betwen the collapse of civil structures or the destruction of the planet" .

Actividad dos: información

Al inicio de la clase posterior, se presentan los resultados en el aula, previamente compilados por el profesor o profesora. Esto genera una discusión abierta, en que se pide que cada participante mencione héroes o heroínas que considere atípicos. A través de este foro abierto, se pretende empezar a identificar los modelos teóricos que responden a la forma en la que entendemos las historias y los cuentos, y cómo los reproducimos y los adaptamos a nuevos medios, como el cine, las series, los videojuegos, etc. También se pretende dar visibilidad a los modelos que tradicionalmente no aparecen: en este caso hablamos de género, pero, como ya he mencionado, también hemos trabajado este modelo de actividad en ciencia ficción y fantasía en relación con razas y especies. Cuando el foco se centra en género, los

\footnotetext{
${ }^{2}$ En castellano: "Un adolescente que se llama Wade tiene el poder de viajar en el tiempo. Descubrió sus poderes cuando tenia 5 años y solo sus padres saben de su don. Wade vive en una granja en Michigan con sus padres, que insisten en que Wade no use sus poderes. Wade siente la necesidad de salvar a la gente y proteger los destinos de las personas."

${ }^{3}$ Manico es una de las regiones del planeta de fantasía presentado por el alumno. Se trata de un lugar desértico.

${ }^{4}$ En castellano: "Djesse, un adolescente humano que vive en Manico [...] Djesse tiene que decidir entre el colapso de las estructuras civiles o la destrucción del planeta."
} 
participantes suelen mencionar ejemplos como Katniss Everdeen de Los Juegos del Hambre, Tormenta de X-Men, la Princesa Leia y Rei de la saga de La Guerra de las Galaxias, Hermione Granger de la saga de Harry Potter, La Capitana Marvel y Wonder Woman.

\section{Objetivo}

El objetivo principal de esta sección de la actividad es comenzar el proceso de interpretación de la información y el cuestionamiento de estereotipos, y un subsiguiente desarrollo de competencias de contraste y comparación. No obstante, el marco teórico que acompaña a la discusión pretende dividir la atención en dos aspectos:

- Conocer teoría literaria basada en narratología y literatura comparada, para lo que:

- Se introducen los modelos de funciones narratológicas de Propp y se aplican a diversos ejemplos

- Se introduce la teoría de la dicotomía naturaleza-cultura de LeviStrauss (Dundes)

- Se introducen las ideas de recepción sincrónica y diacrónica y la necesidad de un estudio contextual

- Ofrecer ejemplos específicos de la fantasía y ficción, para los que se eligen:

- De fantasía:

- El personaje de Eowyn en el libro de El Señor de los Anillos y su adaptación fílmica, mediante un contraste diacrónico y de recepción

- Los grupos de héroes, como los que se encuentran en Memorias de Idhun, donde la existencia de un personaje femenino poderoso convierte la aventura en un triangulo amoroso, escrito por la autora valenciana Laura Gallego.

- De la ciencia ficción o géneros híbridos:

- Ejemplos específicos como La mano izquierda de la oscuridad en que la inexistencia de las diferencias de genero implica una desaparición de los conflictos clásicos de la narratología donde el genero actúa como catalizador del conflicto, de la autora estadounidense Úrsula K. Le Guin.

- La nueva saga de La Guerra de las Galaxias, mediante el conflicto entre la vicealmirante Holdo y Poe, que se desvela a través del punto de vista de Poe y donde se propone una relectura de la idea del 'héroe rebelde'.

Para esta sección, la clase funciona como un foro abierto de participación general y los recursos incluyen una presentación con ejemplos audiovisuales y gráficos específicos, por lo que no se generan resultados ponderables.

\section{Actividad tres: recreación}

La tercera parte de la clase ofrece la opción de reescribir el protagonista de su propuesta $\mathrm{y}$, conscientemente, decidir como sería el nuevo héroe o heroína - si consideran que quieren definirlo por género. La clase se divide en grupos de dos alumnos que tiene que negociar qué línea argumental mantienen y cómo la modifican, tienen un plazo de 15 minutos para proponer un nuevo personaje o modificar el ya 
existente y 5 minutos por grupo para presentarlo al conjunto de la clase. En clases más numerosas, se propone que los grupos sean de un número mayor de alumnos.

\section{Objetivo}

El objetivo principal es la revisión de sus expectativas con un conocimiento incrementado del estado de la cuestión.

\section{Resultados}

Tres de las cuatro historias resultantes proponen mujeres heroínas, mientras que la que originalmente había presentado una héroe trans, la mantiene. Sin embargo, los poderes que les otorgan cambian en las tres heroínas:

- Dos de las heroínas tienen poderes psíquicos: poder leer y manipular la mente de otras personas, y poder crear campos de fuerza

- Una de las heroínas tiene súper fuerza y puede volar. Sus poderes se consideran relacionados con magia, y requiere de una escuela o tutor para desarrollarlos. La protagonista es "a 'normal' 17 year old discovers a secret part of society and is taken away to develop her powers. Unfortunately, danger will reach her before she even knows what her powers are and she is forced to run away to keep her loved ones, the world and herself safe."

\section{TIPOS DE (SÚPER)PODERES}

* visión de rayos $\mathrm{x}$ - poderes psíquicos a fuerza y vuelo

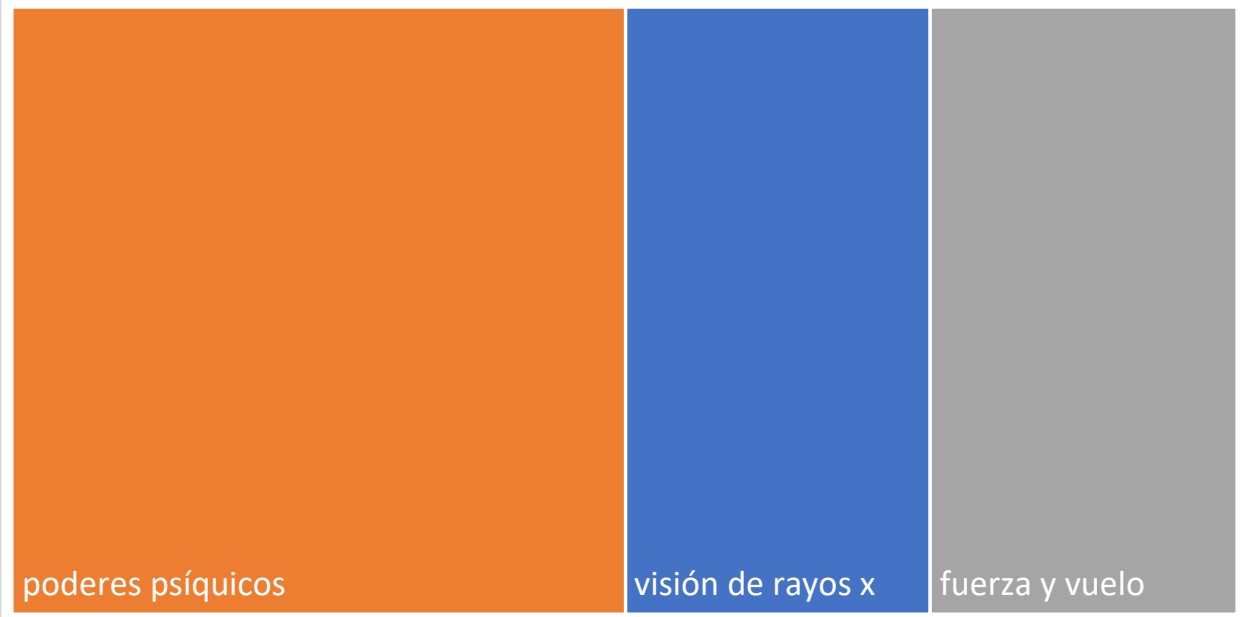

Figura 3: Tipos de poderes de los personajes de la actividad tres

Actividad cuatro: debate

Se pretende cerrar la propuesta con un nuevo foro abierto sobre el proceso: sus decisiones en cuanto a creación y su contexto intertextual. El debate se inicia y se complementa con unos gráficos que hacen referencia a los poderes tradicionales de las súper-heroínas en los cómics propuestos por The Pudding Pool y llevan a formular

\footnotetext{
${ }^{5}$ En castellano: "Una (chica) de 17 años 'normal' descubre una parte secreta de la sociedad y se la llevan para desarrollar sus poderes. Desgraciadamente, le alcanzará el peligro antes de que sepa cuáles son sus poderes y se ve obligada a huir para mantener a salvo a sus seres queridos, al mundo y a sí misma."
} 
preguntas sobre (1) la hipersexualidad de los personajes femeninos (incluso adolescentes, como en Memorias de Idhun o infantiles, como en la saga de Crepúsculo); (2) la percepción de aventura como algo perteneciente a lo que sucede fuera del ámbito doméstico y que implica un conflicto de características bélicas o de grandes gestos (Nikolajeva, 1996, p. 125), unido a (3) la percepción de la verdadera amistad (la camaradería) como solo posible entre hombres y, en relación con estas ideas, (4) lo doméstico como algo invisibilizado y pormenorizado. El objetivo principal es que cada alumno pueda proponer sus preguntas y cuestionar sus propias decisiones creativas a nivel narrativo.

\section{Discusión}

Las conclusiones ofrecen información sobre el doble conflicto: no solo se refleja la escasa presencia de heroínas femeninas de aventuras -el conflicto aparente, al que se responde con una tendencia en alza de su presencia-, sino que aparece el conflicto profundo: cuando las mujeres héroes aparecen, ¿con qué parámetros se miden y que funciones realizan? ¿Se puede generar un nuevo modelo que responda a otros patrones?

\section{DISCUSIÓN GENERAL}

La propuesta didáctica pretende abrir una discusión sobre temas de género trabajando desde la narratología y los modelos conocidos e integrando las expectativas de los participantes. La propuesta responde a la necesidad de incorporar un discurso abierto sobre género en el espacio del aula, donde los alumnos conciban los cambios como una construcción que une lo social y lo comunitario con la identidad individual.

La actividad responde a las competencias que se buscan en el ámbito educativo, donde los alumnos trabajan de diferentes formas (trabajo individual, grupal y en foro abierto), ofreciendo una actividad que invita a una reflexión más profunda del estudio literario. Al generar una historia propia, el alumnado desarrolla el enfoque metacognitivo hacia la crítica literaria, que se puede aplicar en ejercicios posteriores de crítica textual o de recepción, a la vez que la introducción de la escritura creativa ofrece al alumnado un espacio seguro de creación. Se considera que la estructura de esta propuesta también puede ser adaptada al trabajo de otras competencias interculturales, con una selección de materiales pertinentes. En este caso, y al trabajar un enfoque de genero, se ha pretendido incluir no solo la figura femenina como personaje, sino también la figura femenina como creadora literaria.

Quizá la tesis mas importante de esta propuesta pedagógica, tanto para mí como docente como para mis alumnos, es el planteamiento de la pregunta: ¿deberían ser iguales las heroínas a los héroes? Al conocer y cuestionar los compromisos ideológicos, la representación de las heroínas puede visibilizar otro tipo de conflictos y ofrecer soluciones que reconstruyan de un forma distinta la relación con el discurso, la cultura, la sociedad y su ideología.

\section{REFERENCIAS BIBLIOGRÁFICAS}

Arnot, M. y Mac an Ghaill, M. (2006). (Re) contextualising Gender Studies in Education. Schooling in Late Modernity. En Arnot, M. y Mac an Ghaill, M 
(Eds.), The Routledge Falmer Reader in Gender \& Education (pp. 1-14). New York: Routledge.

Bakhtin, M. (1981). The Dialogic Imagination. Austin: University of Texas Press.

Brown, J.S., Collins, A y Duguid P. (1989). Situated Cognition and the Culture of Learning. Educational Researcher, vol. 18. 32-42.

Bruner, J.S. (1996). The Culture of Education. Boston: Harvard University Press.

Burwitz-Melzer, E. (2001) Teaching Intercultural Communicative Competence through Literature. En Byram, M, Nichols, A y Stevens. D (Eds.), Developing Intercultural Competence in Practice (pp. 29-43). Clevedon: Multilingual Matters Ltd.

Campbell, J. (1949). The Hero with a Thousand Faces. Princeton: Princeton University Press.

Campbell, L. M (Ed.) (2014). A Quest Of Her Own: Essays On The Female Hero In Modern Fantasy. Jefferson: McFarland \& Company, Inc.

Davcheva, L. (2003). Learning to Be Intercultural. En Alred. G, Byram, M y Fleming, M (Eds.), Intercultural Experience and Education (pp. 90-109). Clevedon: Multilingual Matters Ltd.

Dundes, A. (1997). Binary Opposition in Myth: The Propp/Lévi-Strauss Debate in Retrospect. Western Folklore. Vol. 56, 39-50. Recuperado de: https://www.jstor.org/stable/1500385

Garcia Carcedo, P. (2011). Escritura Creativa y Competencia Literaria. SEDLL. Lenguaje y Textos. Núm. 33, 49-59. Recuperado de: http://www.sedll.org/sites/default/files/journal/escritura_creativa_y_competencia_literaria._ga rcia_p.pdf

Georgieva, I. (2001). An Approach to Implementing a Cultural Studies Syllabus. En Byram, M, Nichols, A y Stevens. D (Eds.), Developing Intercultural Competence in Practice (pp. 77-92). Clevedon: Multilingual Matters Ltd.

Helford, E.R, et al, (Eds.). (2016) The Woman Fantastic in Contemporary American Media Culture. Jackson: University of Mississippi.

Helford, E.R, et al, (Eds.) (2000). Fantasy Girls: Gender in the New Universe of Science Fiction and Fantasy Television. Boulder: Rowman \& Littlefield Publishers.

Lave, J y Wenger. E. (1991). Situated Learning: Legitimate Peripheral Participation. New York: Cambridge University Press.

Leeds-Hurwitz, W. (2017). Competencias Interculturales: Marco Conceptual y Operativo. UNESCO, Recuperado de: https://unesdoc.unesco.org/ark:/48223/pf000025159.

Leiva Olivencia, J.J., Martín Solbes, V. M., Vila Merino, E. S (Eds.) (2015). Género, Educación y Convivencia. Madrid: Dykinson, S.L.

Messenger Davies, M. (2010) Children, Media and Culture. Issues in Cultural Media Studies. Glasgow: Open University Press. 
Nikolajeva, M. (1996). Children's Literature Comes of Age. New York: Garland Publishing, Inc.

Petit, M. (2002). Pero ¿y Qué Buscan Nuestro Niños En Sus Libros? Lecturas Sobre Lecturas 2, México DF: Consejo Nacional Para La Cultura y Las Artes.

Pritchard, R. (1990). The Effects of Cultural Schemata on Reading Processing Strategies. Reading Research Quarterly, 25, 273-295.

Propp, V. (1968). Morphology of the Folktale. Austin: University of Texas Press.

Shendruk, A. (2017). Analyzing the Gender Representaton of 34,476 Comic Book Characters. The Pudding, Recuperado de: https://pudding.cool/2017/07/comics/

Steinberg, L. (2005). Cognitive and Affective Development in Adolescence. TRENDS in Cognitive Sciences, vol. 9, no. 2, 69-74.

Stephens, J. (1992). Language and Ideology in Children's Fiction. London: Longman.

Tomalin, B y Stempleski, S. (1993). Cultural Awareness. London: Oxford University Press.

Tzvetan, T. (1975). The Fantastic: A Structural Approach to a Literary Genre. Traducido por Howard, R. Ithaca: Cornell University Press. 